\title{
Metastatic status of sentinel lymph nodes in breast cancer determined with photoacoustic microscopy via dual-targeting nanoparticles
}

\author{
Yanfeng Dai ${ }^{1}$, Xiang $\mathrm{Yu}^{1}$, Jianshuang Wei ${ }^{1}$, Fanxin Zeng ${ }^{1}$, Yiran $\mathrm{Li}^{1}$, Xiaoquan Yang ${ }^{1}$, Qingming $L \mathrm{~L}^{1,2}$ and \\ Zhihong Zhang $\mathbb{1}^{1,2}$
}

\begin{abstract}
Detection of sentinel lymph nodes (SLNs) is critical to guide the treatment of breast cancer. However, distinguishing metastatic SLNs from normal and inflamed lymph nodes (LNs) during surgical resection remains a challenge. Here, we report a CD44 and scavenger receptor class B1 dual-targeting hyaluronic acid nanoparticle (5K-HA-HPPS) loaded with the near-infra-red fluorescent dye DiR-BOA for SLN imaging in breast cancer. The small sized $(\sim 40 \mathrm{~nm})$ self-assembled 5K-HA-HPPSs accumulated rapidly in the SLNs after intradermal injection. Compared with normal popliteal LNs (N-LN), there were $\sim 3.2$-fold and $\sim 2$.4-fold increases in fluorescence intensity in tumour metastatic SLNs (T-MLN) and inflamed LNs (Inf-LN), respectively, $6 \mathrm{~h}$ after nanoparticle inoculation. More importantly, photoacoustic microscopy (PAM) of 5KHA-HPPS showed a significantly distinct distribution in T-MLN compared with N-LN and Inf-LN. Signals were mainly distributed at the centre of T-MLN but at the periphery of N-LN and Inf-LN. The ratio of PA intensity (R) at the centre of the LNs compared with that at the periphery was $5.93 \pm 0.75$ for T-MLNs of the 5K-HA-HPPS group, which was much higher than that for the Inf-LNs $(R=0.2 \pm 0.07)$ and N-LNs $(R=0.45 \pm 0.09)$. These results suggest that 5K-HA-HPPS injection combined with PAM provides a powerful tool for distinguishing metastatic SLNs from pLNs and inflamed LNs, thus guiding the removal of SLNs during breast cancer surgery.
\end{abstract}

\section{Introduction}

Breast cancer is the most commonly diagnosed cancer and the leading cause of cancer-related mortality among women ${ }^{1}$. Globally, there were $\sim 2.1$ million newly diagnosed cases of breast cancer in 2018 alone, accounting for $\sim 25 \%$ of cancer cases in women ${ }^{2}$. Despite advances in diagnosis and treatment, metastatic breast cancer, particularly hormone receptor-positive cancer, is considered incurable ${ }^{3,4}$. To balance the reduction in pain and benefits

\footnotetext{
Correspondence: Qingming Luo (qluo@hainanu.edu.cn) or

Zhihong Zhang (czyzzh@mail.hust.edu.cn)

'Britton Chance Center and MOE Key Laboratory for Biomedical Photonics, School of Engineering Sciences, Wuhan National Laboratory for

Optoelectronics-Huazhong University of Science and Technology, Wuhan, Hubei 430074, China

${ }^{2}$ School of Biomedical Engineering, Hainan University, Haikou, Hainan 570228, China

These authors contributed equally: Yanfeng Dai, Xiang Yu, Jianshuang Wei
}

of an extended lifespan against the harm caused by treatment, a rational treatment programme should be based on tumour-node-metastasis staging ${ }^{5}$. In recent years, immunological surveillance of normal lymph nodes (LNs) has gradually enabled the replacement of complete axillary LN dissection by sentinel LN (SLN) biopsy (SLNB) as a means to accurately stage $\mathrm{LNs}^{6}$. Although SLNB in breast cancer has been indicated to be effective in reducing postoperative morbidity ${ }^{7}$, this procedure has some disadvantages. The standard SLNB method in a clinical setting involves injection of a technetium-labelled nanocolloid preoperatively into the breasts around the tumour, which increases patient concerns about further radiation exposure and reoperation ${ }^{8}$. Moreover, subsequent histological analysis of LN metastasis after SLNB is time-consuming and somewhat subjective. In addition, SLN dissection may cause complications such as

\section{(c) The Author(s) 2020}

(c) Open Access This article is licensed under a Creative Commons Attribution 4.0 International License, which permits use, sharing, adaptation, distribution and reproduction in any medium or format, as long as you give appropriate credit to the original author(s) and the source, provide a link to the Creative Commons license, and indicate if changes were made. The images or other third party material in this article are included in the article's Creative Commons license, unless indicated otherwise in a credit line to the material. If material is not included in the article's Creative Commons license and your intended use is not permitted by statutory regulation or exceeds the permitted use, you will need to obtain permission directly from the copyright holder. To view a copy of this license, visit http://creativecommons.org/licenses/by/4.0/. 
lymphedema, shoulder dysfunction and numbness ${ }^{9,10}$. Therefore, the development of a new approach to accurately identify LN metastases intraoperatively will help surgeons choose appropriate treatment plans with improved effects and minimise the complications caused by unnecessary LN removal.

Several non-radiative approaches that can rapidly predict the metastatic status of SLNs have been developed. They include fluorescence imaging using a targeted fluorescent agent ${ }^{11}$, contrast-enhanced ultrasound using microbubbles ${ }^{12}$, nanoparticle-enhanced MRI ${ }^{13,14}$ and imaging by surface-enhanced Raman scattering ${ }^{15}$. Because these agents are mainly taken up by macrophages in LNs, these methods distinguish between tumours metastatic SLNs (T-MLN) and inflamed LNs (Inf-LN) according to the lower signal intensity of metastatic SLNs. However, metastatic LNs with a low signal intensity are less likely to be detected intraoperatively under a blurred visual field. Unfortunately, this drawback is further amplified when the lymphatic tissue is entirely occupied by tumour tissue at the late stage or when the afferent lymphatic vessels are blocked by metastatic tumours. Therefore, there is an urgent need to develop a new strategy based on the characteristics of the metastatic tumour itself to discriminate normal LNs (N-LN) and Inf-LN from SLNs in breast cancer. To selectively target breast cancer cells in SLNs, two factors must be taken into consideration. One is that the tracer agent should rapidly migrate to LNs by either a passive targeting mechanism, an active targeting mechanism, or both. The other factor is that the tracer agent should contain a specific ligand for the selective targeting of breast cancer cells.

Hyaluronic acid (HA), a linear mucopolysaccharide possessing a large number of negatively charged carboxyl groups, is an essential component of the extracellular matrix that is generated naturally in all living organisms ${ }^{16}$. Due to its outstanding biocompatibility and non-immunogenicity, HA and its derivatives have been clinically used as medical products for tissue repair and regeneration for several decades ${ }^{17}$. HA is also a well-known ligand of CD44, which is overexpressed in breast cancer ${ }^{18}$; thus, HA can serve as an ideal candidate for designing novel HA-based nanocarriers that selectively target breast cancer cells. However, the turnover of CD44 is modulated by occupancy with HA, which can easily lead to saturation ${ }^{19}$. In addition, glycosylation of CD44 negatively regulates its ability to recognise HA and inhibits CD44-mediated uptake efficiency ${ }^{20}$, suggesting that only HA-mediated CD44 targeting of tumour cells is insufficient to acquire images with a high signal-to-noise ratio. Therefore, a dual receptortargeted drug delivery system will be helpful to target breast cancer cells more effectively.
Particle size is the most important factor in nanoparticle migration to LNs. Although the optimal size range remains a matter of debate, it is generally considered that ultrasmall nanoparticles $(10-50 \mathrm{~nm})$ show rapid uptake and long retention times in the lymphatic system ${ }^{21,22}$. Moreover, it is favourable to transport negatively charged nanoparticles into the lymphatic system in the interstitial fluid due to the negative charge on glycosaminoglycans in the extracellular matrix $^{23}$. Previously, we developed a high-density lipoprotein (HDL)-mimicking peptide-phospholipid scaffold (HPPS) with hydrodynamic diameters of $10-30 \mathrm{~nm}^{24}$ that can migrate to LNs by size-dependent passive transport ${ }^{21,25}$. Moreover, HPPS nanoparticles can target scavenger receptor class B type 1 (SR-B1), which is expressed on breast cancer cells ${ }^{26}$. Herein, we developed a novel dualtargeting nanoparticle by inserting the negatively charged HA-1,2-dimyristoyl phosphatidylethanolamine (DMPE) into a single-layer phospholipid of HPPS, denoted HAHPPS. Importantly, this dual-targeting nanoparticle has a phospholipid monolayer shell containing DiR-BOA, a nearinfra-red (NIR) fluorophore, which can be used for both fluorescence imaging and photoacoustic microscopy $(\mathrm{PAM})^{27,28}$. We can continuously monitor changes in the DiR-BOA fluorescence signal of LNs in a mouse model (e.g. inflammatory or tumour model) by using a wide-field fluorescent imaging system that has the advantages of sensitivity, convenience and non-invasiveness. By providing deep penetration and high spatial resolution, PAM has great potential for the $3 \mathrm{D}$ visualisation of photoacoustic signal distribution in intact LNs. In this proof-of-concept study (Fig. 1), we hypothesised that HA-HPPS combined with fluorescence/photoacoustic imaging can enable the visualisation of SLNs in a rapid and prolonged manner through size- and charge-dependent passive and active targeting of both CD44 and SR-B1, which can guide the removal of SLNs from patients during breast cancer surgery.

\section{Results}

Synthesis and characteristics of the CD44 and SR-B1 dualtargeted lipid nanoparticles

To synthesise CD44 and SR-B1 dual-targeted nanoparticles, we first synthesised 5K-HA-DMPE or 15K-HADMPE using 5-kDa HA- or 15-kDa HA-conjugated DMPE (Supplementary Fig. S1a). After purification, the chemical structures of HA, DMPE and HA-DMPE were determined by ${ }^{1} \mathrm{H}$ NMR spectroscopy. The chemical shifts of the protons of the terminal methyl $(0.9-1.0 \mathrm{ppm})$ and methylene $(1.26 \mathrm{ppm})$ groups of DMPE and the N-acetyl group (1.93 ppm) of HA were used to determine HADMPE synthesis (Supplementary Fig. S1b). Then, HADMPE was inserted into a single-layer phospholipid of HPPS to form HA-HPPS, and the final product was purified using a fast protein liquid chromatography (FPLC) system. The FPLC profile, in which absorption 
a

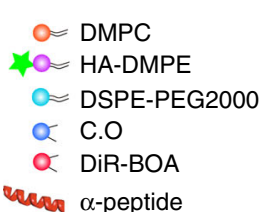

b
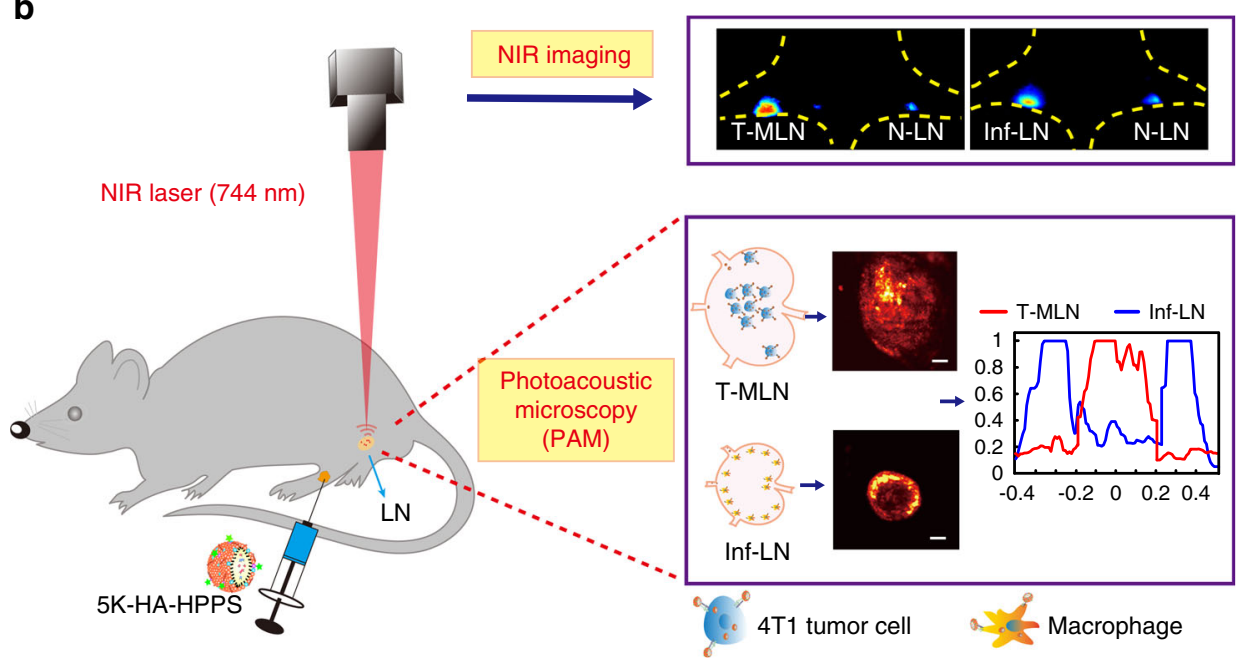

Fig. 1 Design of dual-modality HA-HPPS nanoparticles for mapping sentinel lymph nodes in breast cancer. a Components and structures of the CD44 and SR-B1 dual-targeting HA-HPPS nanoparticles. b Dual-modality fluorescence and photoacoustic imaging of HA-HPPS in SLNS, which includes near-infra-red (NIR) fluorescence imaging for long-term monitoring of the accumulation and retention of HA-HPPS in SLNs and photoacoustic microscopy (PAM) for intraoperative determination of the metastatic status of SLNs in breast cancer curves at $280 \mathrm{~nm}$ and $700 \mathrm{~nm}$ represented HA-HPPS and DiR-BOA, respectively (Fig. 2a), showed that two main narrow peaks at retention times of 67.4 and $70 \mathrm{~min}$ represented 5K-HA-HPPS and 15K-HA-HPPS, respectively. Transmission electron microscopy (TEM) clearly showed that 5K-HA-HPPS and 15K-HA-HPPS had uniform spherical morphologies (Supplementary Fig. S2a). Dynamic light scattering (DLS) indicated that 5K-HAHPPS and 15K-HA-HPPS were $\sim 43$ and $\sim 38 \mathrm{~nm}$ in size, respectively (Supplementary Fig. S2b). Notably, these sizes were smaller than those of the $5 \mathrm{~K}-\mathrm{HA}$ emulsion $(5 \mathrm{~K}$ HA-e, $\sim 57.6 \mathrm{~nm}$ ) and 15K-HA emulsion (15K-HA-e, $\sim 53.6 \mathrm{~nm}$ ) (Supplementary Fig. S2c). In addition, the zeta potential profiles indicated that 5K-HA-HPPS and $15 \mathrm{~K}-$ HA-HPPS carried slight negative charges $(-7.2 \pm 0.6$ and $-5.6 \pm 0.6 \mathrm{mV}$, respectively) (Supplementary Fig. S2d). The 5K-HA-HPPS and 15K-HA-HPPS absorption spectra both showed a maximum absorption peak at $748 \mathrm{~nm}$ (Fig. 2b). 5K-HA-HPPS and 15K-HA-HPPS fluorescence imaging were well correlated with the presented DiR-BOA concentrations (Fig. 2c). Next, we verified that the PA signals of the 5K-HA-HPPS solutions with different DiRBOA concentrations were linearly dependent on the concentration $\left(R^{2}=0.9969\right)$ from 31.25 to $500 \mu \mathrm{g} \mathrm{ml}^{-1}$ (Fig. 2d). The PA signal of 5K-HA-HPPS was comparable to that of the FDA-approved NIR-PA contrast agent ICG at the same concentration at $744 \mathrm{~nm}$, but the PA signal of ICG at $800 \mathrm{~nm}$ was much stronger than that of 5K-HAHPPS and 1.4 times that of 5K-HA-HPPS at $744 \mathrm{~nm}$ (Fig. 2e). To verify the stability of HA-HPPS, the HA-HPPS core loaded with DiR-BOA was additionally labelled with fluorescein isothiocyanate (FITC) to form the duallabelled FITC-HA-HPPS(DiR-BOA). Seminative SDS polyacrylamide gel electrophoresis (PAGE) indicated that both 5K-HA-HPPS and 15K-HA-HPPS were stable in $10 \%$ mouse plasma at $37^{\circ} \mathrm{C}$, even after continuous incubation for $24 \mathrm{~h}$. As a control, 10\% Triton-X 100 disrupted the structure of HA-HPPS, resulting in unrestricted diffusion of DiR-BOA fluorescence signals (Supplementary Fig. S2e). Finally, to assess the cytotoxicity of the nanoparticles, we examined the viability of RAW264.7 cells after incubation with different concentrations of HAHPPS for 24h using the (3-(4,5-dimethylthiazol-2-yl)-5(3-carboxymethoxyphenyl)-2-(4-sulfophenyl)-2H-tetrazolium) (MTS) assay. As shown in Supplementary Fig. S2f, $\sim 100 \%$ of the RAW264.7 cells survived with a wide 


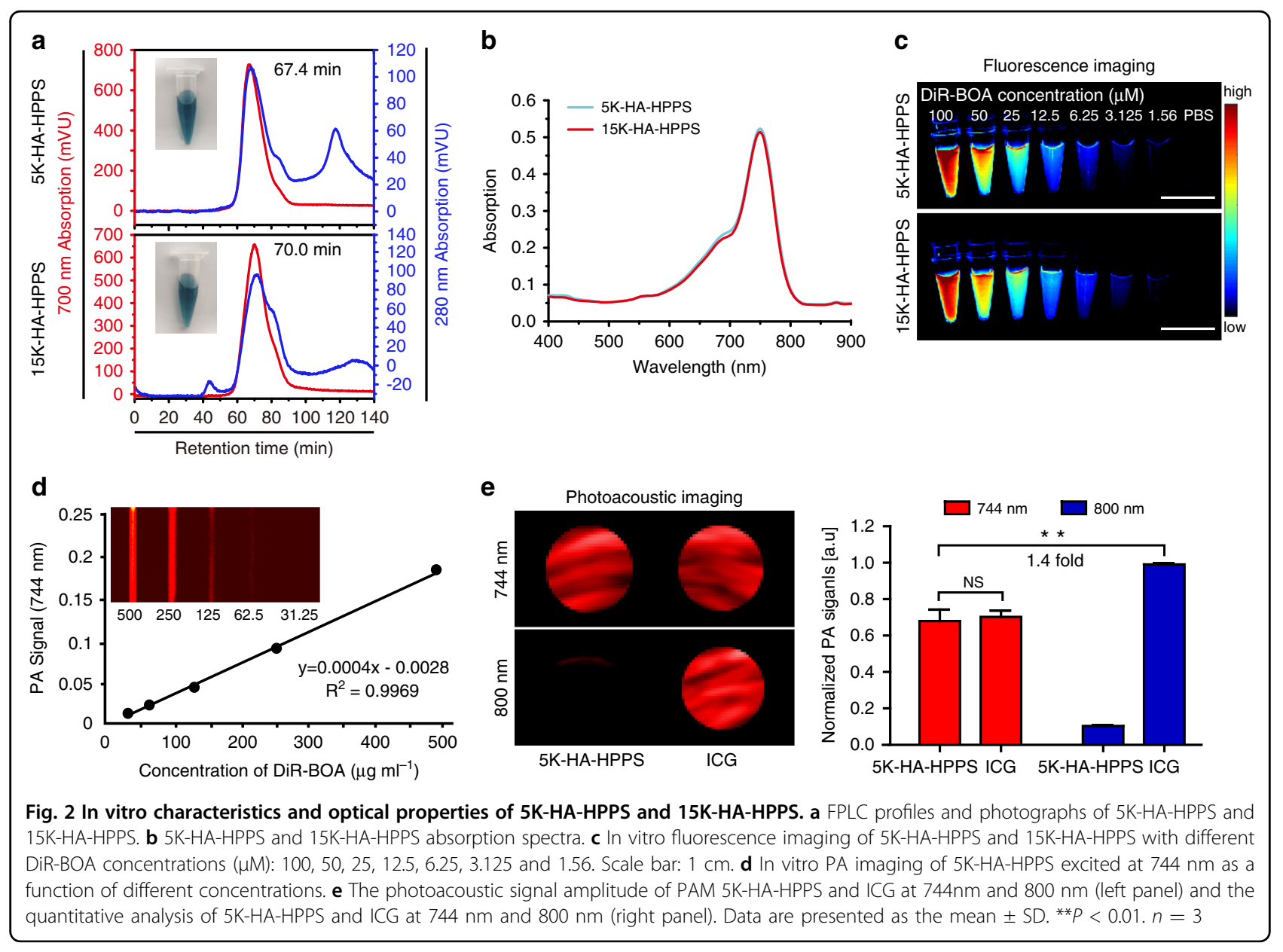

range of HA-HPPS concentrations (peptide concentrations: $1-20 \mu \mathrm{M})$ after $24 \mathrm{~h}$ of incubation. These results suggested that the HA-based nanoparticles have good biocompatibility for in vivo applications.

\section{K-HA-HPPS migrates to LNs rapidly and efficiently}

To explore the effects of HA-HPPSs of similar sizes and charges on the non-invasive imaging of LNs in vivo, we investigated the lymphatic trafficking ability of HA-HPPS in albino C57BL/6 mice. Following intradermal injection of HA-HPPS, HPPS, 5K-HA-e, or 15K-HA-e into the hind footpads of mice, wide-field fluorescence imaging was performed to record the migration and retention of the imaging tracers at different time points after different exposure times (30 s in Fig. 3a and $10 \mathrm{~s}$ in Supplementary Fig. S3). As shown in Fig. 3a, 5K-HA-HPPS migrated rapidly to popliteal LNs (pLNs), showing a strong fluorescence signal within $10 \mathrm{~min}$. The mean fluorescence intensity (MFI) of DiR-BOA in pLNs gradually increased with time and reached a maximum at $6 \mathrm{~h}$ and was strong even at $12 \mathrm{~h}$. However, pLNs injected with HPPS or 5K-HA-e presented only relatively weak fluorescence signals $1 \mathrm{~h}$ after injection. The MFIs of DiR-BOA in 5K-HA-HPPS were $\sim 3.0$ - and $\sim 2.3$-fold greater than those of HPPS and 5KHA-e at $6 \mathrm{~h}$ and $\sim 3.2$ - and $\sim 2.5$-fold greater at $12 \mathrm{~h}$, respectively (Fig. $3 \mathrm{~b}$ ). This result was confirmed by in vitro imaging of excised pLNs at $12 \mathrm{~h}$ (Fig. 3c). Interestingly, all sciatic LNs in the three groups showed similar signal intensities. These results suggested that 5K-HA-HPPS preferentially accumulated in pLNs and migrated to secondary LNs. When the exposure time was reduced to $10 \mathrm{~s}$, clear fluorescence signals of DiR-BOA in the pLNs of the 5K-HA-HPPS group remained detectable at significantly stronger levels than those of the control groups (Supplementary Fig. S3). However, the fluorescence signals of DiRBOA in the pLNs of the 15K-HA-HPPS group were very weak (Supplementary Fig. S4a). Moreover, there were no significant differences in the MFIs of DiR-BOA among the 15K-HA-HPPS, HPPS and 15K-HA-e groups (Supplementary Fig. S4b, c). These results indicated that 5K-HA-HPPS, rather than 15K-HA-HPPS, migrates rapidly and efficiently to LNs and can serve as a good LN imaging agent.

\section{Dual-targeting ability of 5K-HA-HPPS in 4T1 cells}

Having confirmed the ability of 5K-HA-HPPS to target LNs, we evaluated the ability of 5K-HA-HPPS to target 


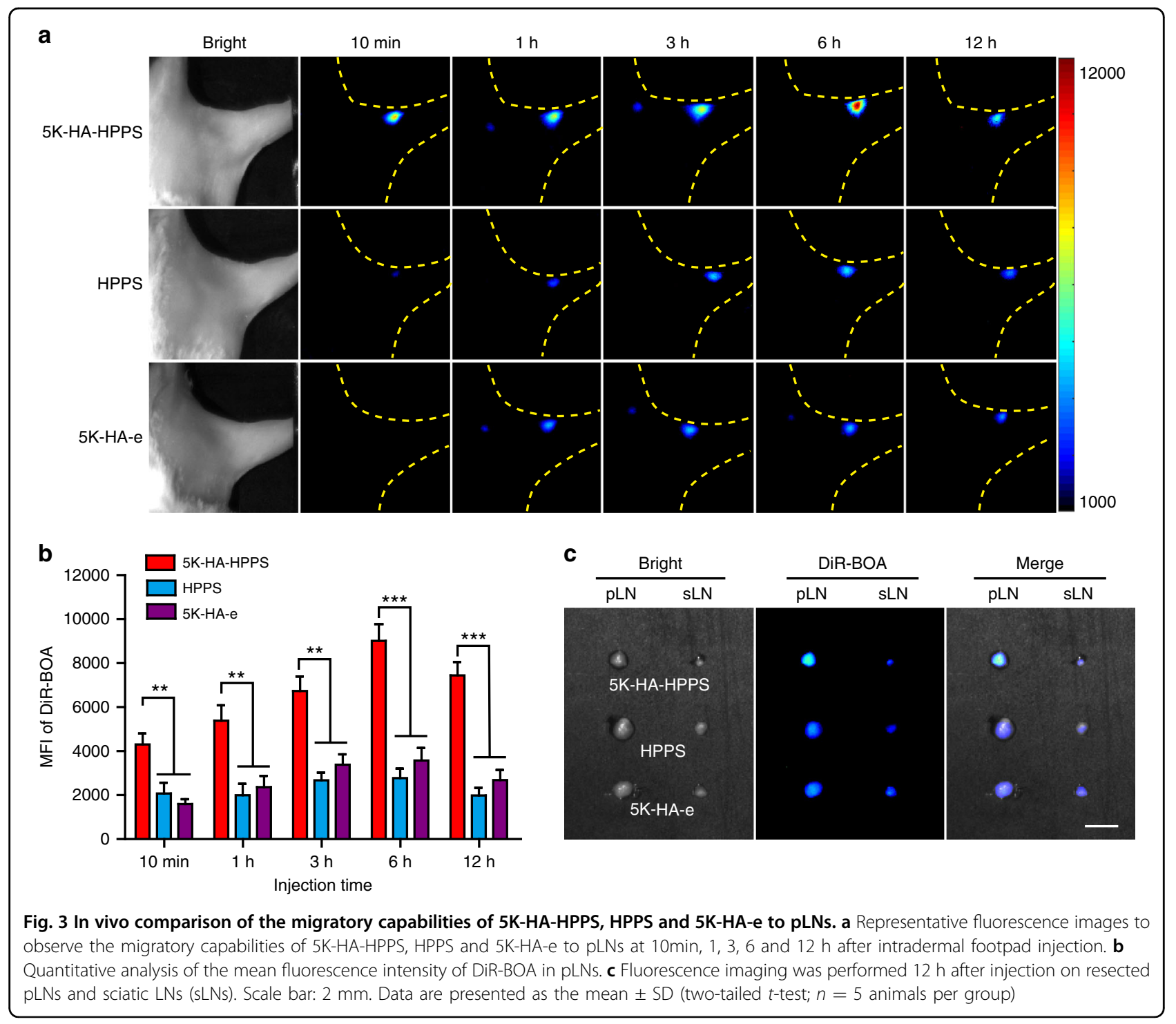

4T1 cells, which highly express SR-B1 and CD44, using flow cytometry (FCM) and confocal imaging. After 0.5 and $3 \mathrm{~h}$ of incubation, FCM showed that 4T1 cells took up nanoparticles in a concentration-dependent manner, and the MFI of DiR-BOA in 4T1 cells increased with incubation time (0.5-3 h) (Fig. 4a, b). The MFI of DiR-BOA in $4 \mathrm{~T} 1$ cells treated with dual-targeted 5K-HA-HPPS was higher than that of single-targeted HPPS and 5K-HA-e, with a 6.3- and 2.4-fold difference, respectively, at a high concentration (20 $\mu \mathrm{M}$ DiR-BOA) after $0.5 \mathrm{~h}$ of incubation (Fig. 4a). Interestingly, at DiR-BOA concentrations of $>2 \mu \mathrm{M}(2-20 \mu \mathrm{M})$, the uptake of $5 \mathrm{~K}-\mathrm{HA}-\mathrm{e}$ by $4 \mathrm{~T} 1$ cells was stronger than that of HPPS (Fig. 4a, b). This effect may be due to the overexpression of CD44 on 4T1 cells and highly efficient uptake of 5K-HA-e. Moreover, after $3 \mathrm{~h}$ of incubation, confocal imaging demonstrated that the nanoparticles were distributed mainly in the cytoplasm, and the fluorescence intensity of $4 \mathrm{~T} 1$ cells treated with 5K-HA-HPPS was significantly stronger than that of HPPS- and 5K-HA-e-treated cells (Fig. 4c). In addition, we detected the uptake of 5K-HA-HPPS by B16F10 melanoma cells, CT26 colon tumour cells and E0771 breast cancer cells, which also express CD44 and SR-B1. FCM showed that E0771 cells effectively took up 5K-HAHPPS with a similar ability as 4T1 cells, and both B16 and CT26 cells also had a strong uptake of 5K-HA-HPPS (Supplementary Fig. S5). To further demonstrate that the endocytosis of nanoparticles by $4 \mathrm{~T} 1$ cells is mediated by the CD44 and SR-B1 receptors, we designed a competitive inhibition experiment by treating the 4T1 cells with an excessive amount of free HDL protein and/or HA, which blocked the SR-B1 and CD44-mediated uptake of 5K-HAHPPS, respectively. FCM showed that excessive HDL protein and HA dramatically inhibited the internalisation 


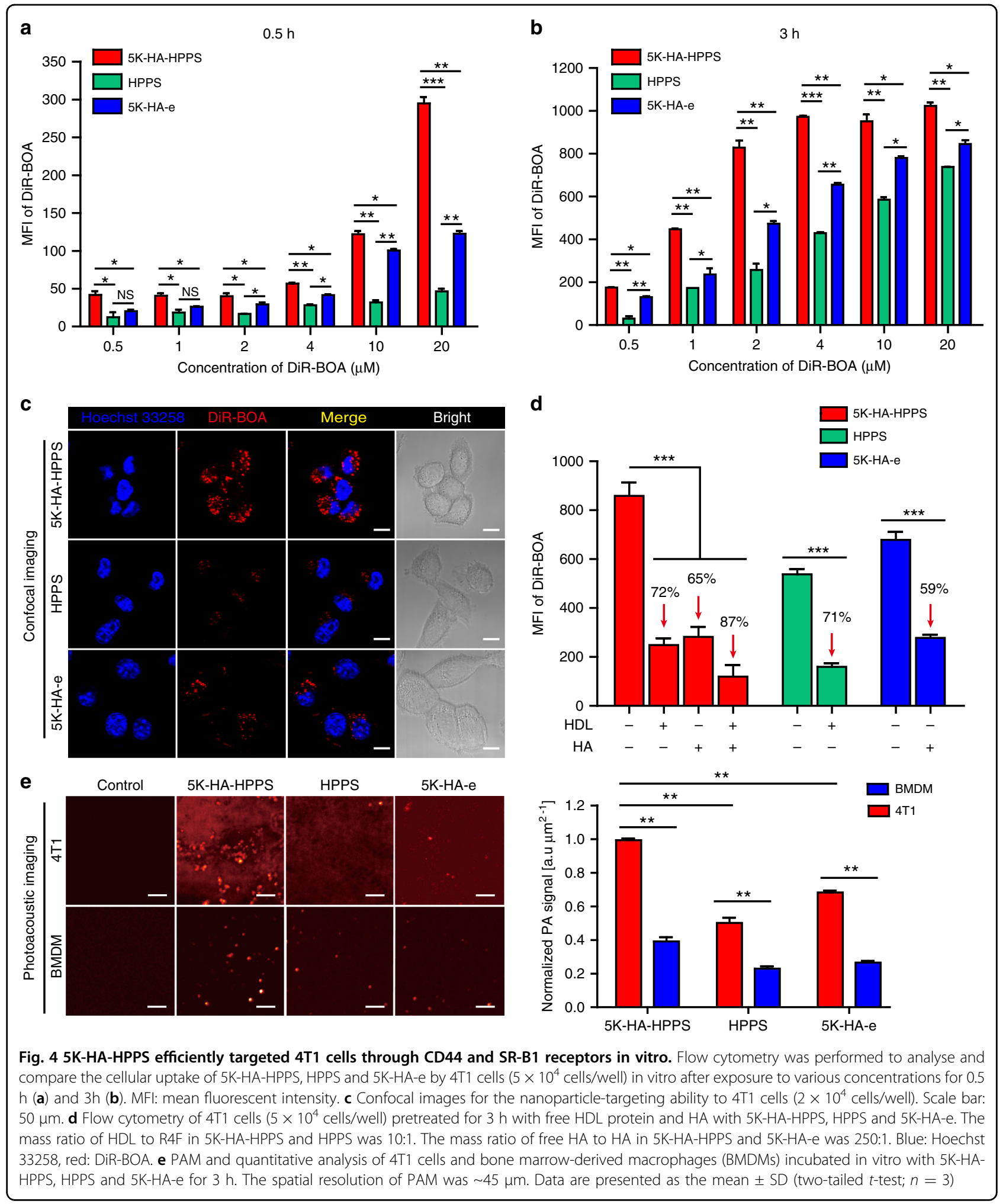

of HPPS (71\% decrease) and 5K-HA-e (59\% decrease) by 4T1 cells (Fig. 4d). In addition, when excessive HDL protein and HA were added simultaneously, the uptake of $5 \mathrm{~K}-\mathrm{HA}-\mathrm{HPPS}$ decreased by $87 \%$ in $4 \mathrm{~T} 1$ cells.
Furthermore, we compared the uptake efficiency of 5KHA-HPPS by 4T1 cells, bone marrow-derived macrophages (BMDMs) and RAW264.7 cells. The flow cytometry data showed that the efficiency of 5K-HA-HPPS 
uptake by $4 \mathrm{~T} 1$ cells was much higher than that of BMDM cells, with a 3.5 - and 1.8 -fold difference at $2 \mu \mathrm{M}$ and $10 \mu \mathrm{M}$ DiR-BOA after $0.5 \mathrm{~h}$ of incubation and 3.9- and 2.8 -fold after $3 \mathrm{~h}$ of incubation, respectively (Supplementary Fig. S6a). In addition, after $3 \mathrm{~h}$ of incubation, confocal imaging demonstrated that the fluorescence intensity of 4T1 cells treated with 5K-HA-HPPS was significantly stronger than that of BMDMs, RAW264.7 cells and bone marrow-derived dendritic cells (BMDCs) (Supplementary Fig. S6b). We used PAM to compare the uptake ability of 5K-HA-HPPS, HPPS and 5K-HA-e by $4 \mathrm{~T} 1$ tumour cells and BMDMs after incubation for $3 \mathrm{~h}$ in vitro. As shown in Fig. 4e, the PAM signal in 4T1 cells was stronger than that in BMDM cells, and the PAM signal of 4T1 cells incubated with 5K-HA-HPPS was much stronger than that in the HPPS and 5K-HA-e groups. Collectively, these results indicated that dual-targeting of SR-B1- and CD44mediated endocytosis can explain the increased cellular uptake efficiency of 5K-HA-HPPS by $4 \mathrm{~T} 1$ cells.

Next, the in vivo ability of 5K-HA-HPPS to target metastatic 4T1 cells was examined in a LN metastasis tumour model, which was established by hock inoculation of 4T1-tfRFP cells in the left legs of BALB/c mice (Fig. 5a). Three weeks after tumour inoculation, primary tumours had developed (Fig. 5b). Compared with N-LNs, T-MLNs were evidenced by their enlarged size and increased fluorescence intensity of the excised LNs (Fig. 5b). Confocal imaging of the T-MLN sections indicated that the 4T1-tfRFP cells were distributed mainly in the inner part of the LNs, whereas only a few tumour cells were distributed in the periphery (Fig. 5b). Then, FITC-labelled nanoparticles were injected into the primary tumours, and LNs were removed $6 \mathrm{~h}$ later. Overall, T-MLNs had bright fluorescence signals from both the tfRFP protein and FITC, indicating that the 4T1-tfRFP cells metastasised into the SLNs and that the nanoparticles accumulated in the SLNs (Fig. 5c). Confocal imaging of LN sections indicated that there were many 4T1-tfRFP cells inside tumour-draining LNs. More importantly, FITC-labelled 5K-HA-HPPS colocalized with the majority of the 4T1tfRFP cells in SLNs, whereas tumour cells labelled with FITC-HPPS were rarely observed (Fig. 5d). Thus, 5K-HAHPPS can effectively target $4 \mathrm{~T} 1$ cells by dual-targeting CD44 and SR-B1 in vitro and in vivo.

\section{In vivo NIR fluorescence imaging for the detection of 4T1 cells in metastatic LNs}

Inspired by the fact that 5K-HA-HPPS was found to effectively target SLNs and metastatic $4 \mathrm{~T} 1$ cells, we proposed that 5K-HA-HPPS can distinguish between metastatic and normal LNs. A LN metastasis model of 4T1 breast tumours was established as described above, but we did not use 4T1-tfRFP due to the slow growth of this cell line and the period required to establish the model. After injecting 5K-HA-HPPS, HPPS or 5K-HA-e into primary tumours and contralateral subcutaneous injection, widefield fluorescence imaging was performed to record the migration and retention of imaging tracers at different timepoints. As shown in Fig. 6a, there was a dramatically higher tracer uptake in popliteal T-MLNs compared with contralateral N-LNs at all timepoints, and the DiR-BOA signal intensity from T-MLNs in the 5K-HA-HPPS group was significantly greater than the signal intensities in the HPPS and 5K-HA-e groups. Quantitative analysis indicated that the accumulation of 5K-HA-HPPS in T-MLNs was 3.3- and 4.25-fold higher than that in the contralateral $\mathrm{N}-\mathrm{LNs}$ at 6 and $24 \mathrm{~h}$, respectively (Fig. 6b). Moreover, the MFI of DiR-BOA in the T-MLNs of the 5K-HA-HPPS group was 2.16- and 2.45-fold higher than that in the HPPS and 5K-HA-e groups at $6 \mathrm{~h}$, respectively, and 3.38and 3.36 -fold higher than that at $24 \mathrm{~h}$, respectively (Fig. $6 \mathrm{~b})$. It is noteworthy that the T-MLNs of the 5K-HAHPPS group had a strong DiR-BOA fluorescence signal for $24 \mathrm{~h}$, and the contralateral N-LNs showed a similar trend but with much lower signal intensity. There was no significant difference in terms of the fluorescence intensity of DiR-BOA between T-MLNs and contralateral NLNs at $24 \mathrm{~h}$ in the HPPS and 5K-HA-e groups (Fig. 6b). This may be due to the uptake of 5K-HA-HPPS by metastatic tumour cells in LNs and the subsequent long period of retention in LNs. Ex vivo fluorescence imaging of 5K-HA-HPPS in resected LNs confirmed the significant difference in fluorescence intensity of DiR-BOA between ipsilateral T-MLNs and contralateral N-LNs (Fig. 6c). The extensive infiltration of tumour cells to the ipsilateral node was also confirmed by the enlarged size of the node (Fig. 6c) and H\&E staining of pLNs (Fig. 6d). We also established an orthotopic 4T1 breast tumour model to assess the ability of 5K-HA-HPPS to map metastatic LNs. In addition to primary tumours (yellow arrows), strong fluorescence intensity was seen in the brachial and axillary LNs of mice (yellow circles). Whole-body fluorescence imaging confirmed higher DiR-BOA fluorescence intensities for brachial and axillary LNs in the 5K-HAHPPS group than in the HPPS group in vivo and ex vivo (Supplementary Fig. S7a, b). These results indicated that dual-targeting by $5 \mathrm{~K}$-HA-HPPS permits the mapping of SLNs and discriminates between metastatic and normal LNs through enhanced uptake and retention.

\section{Inflammation-induced LNs highlighted by 5K-HA-HPPS}

To determine the ability of 5K-HA-HPPS to distinguish between inflamed LNs (Inf-LNs) and N-LNs, we established an inflammatory LN model by intra-lymph node injection of lipopolysaccharide (LPS) into left pLNs $(n=$ 5). As shown in Fig. 7a, due to inflammatory stimulation, left Inf-LNs showed an obviously higher level of tracer accumulation than the contralateral N-LNs in the 


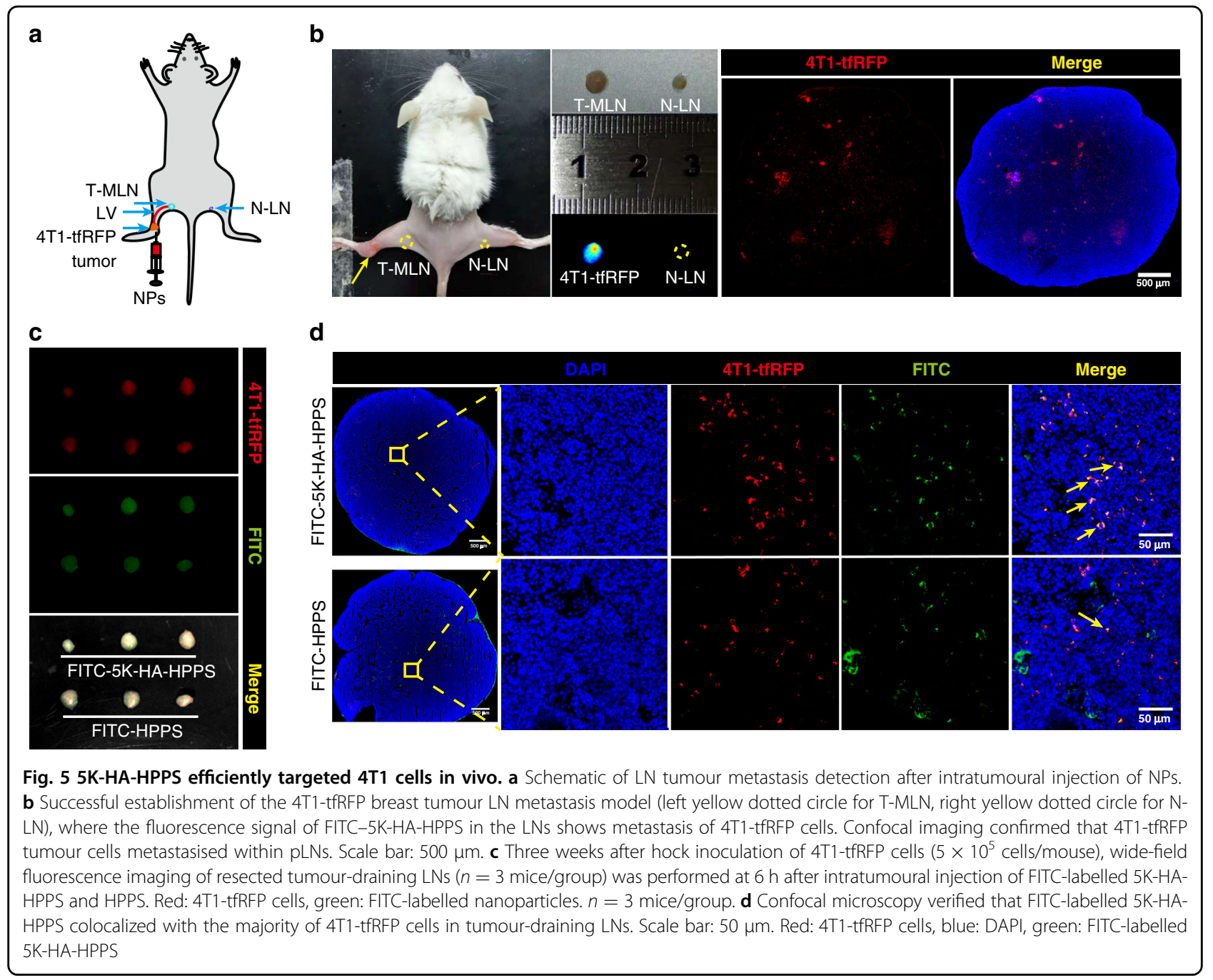

5K-HA-HPPS group. Quantification of the fluorescence images showed that the MFI of DiR-BOA in the left InfLNs was 2.7- and 2.5-fold higher than that in the contralateral N-LNs at 6 and $24 \mathrm{~h}$, respectively (Fig. 7b) but was weaker than that in T-MLNs (Supplementary Fig. S8a). Moreover, the MFI of DiR-BOA in the 5K-HAHPPS group was 2.43- and 2.5-fold higher than that in the left Inf-LNs of the HPPS and 5K-HA-e groups at $6 \mathrm{~h}$, respectively. Although left Inf-LNs showed somewhat higher fluorescence intensity than the contralateral NLNs in the HPPS and 5K-HA-e groups, no significant difference was found, except at 6 and $12 \mathrm{~h}$ (Fig. 7a, b). Ex vivo fluorescence images $24 \mathrm{~h}$ after injection showed that LPS-induced Inf-LNs were larger than N-LNs and had stronger signals, particularly in the 5K-HA-HPPS group (Fig. 7c). Immunofluorescence imaging of Inf-LNs showed that $5 \mathrm{~K}-\mathrm{HA}-\mathrm{HPPS}$ is mainly taken up by macrophages $\left(\mathrm{F} 4 / 80^{+}\right)$and partially by lymphatic endothelial cells (LECs, Lyve- $1^{+}$) and dendritic cells (DCs, CD11 ${ }^{+}$) but rarely taken up by $\mathrm{B}$ cells $\left(\mathrm{B}^{2} 20^{+}\right)$and $\mathrm{T}$ cells $\left(\mathrm{CD}^{+}\right)$
(Supplementary Fig. S8b). These results demonstrated that 5K-HA-HPPS is an effective tool for the detection of inflammation-induced LNs through enhanced signal intensity.

\section{In vivo photoacoustic imaging for predicting the metastatic status of SLNs}

The preceding results demonstrated that both metastatic and inflamed LNs exhibit significantly enhanced nanoparticle uptake, so it is not easy to distinguish between these LNs by fluorescence imaging alone. PAM is a rapidly developing imaging technology for both preclinical and clinical applications. Based on differences in absorbance and the inherent advantages of high-contrast optical imaging and deep ultrasound imaging depth, PAM has been implemented for multiscale imaging from acoustic resolution to optical resolution ${ }^{29-31}$. Given that DiR-BOA can be used for PAM as well as fluorescence imaging, we anticipated that 5K-HA-HPPS(DiR-BOA) can effectively distinguish metastatic SLNs from 


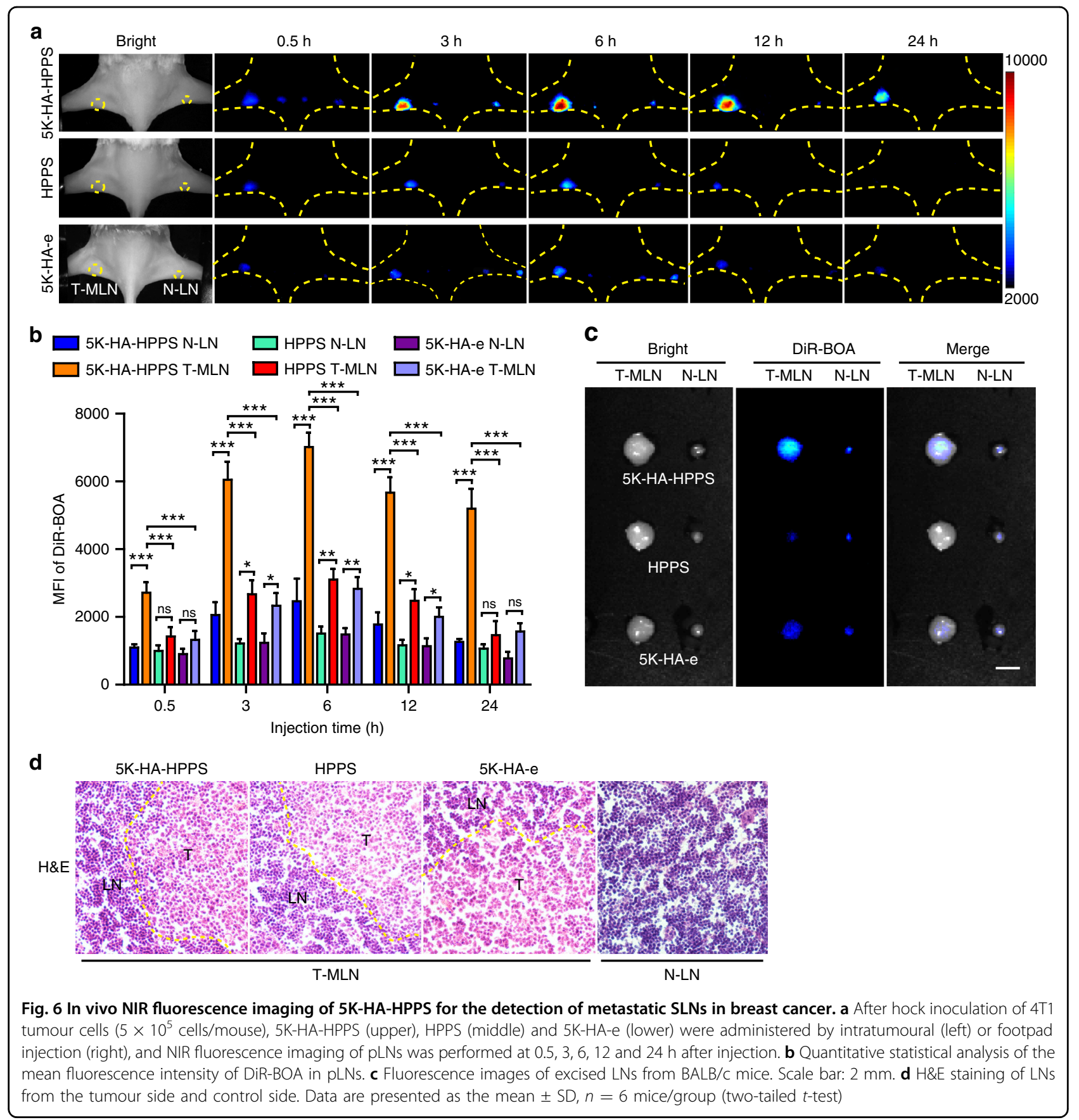

inflammatory LNs according to the spatial distribution of the PA signal. We performed in vivo PAM for exposed pLNs $6 \mathrm{~h}$ after hock injection of nanoparticles. As shown in Fig. 8a, there were strong PA signals at the peripheries of N-LNs and Inf-LNs in the 5K-HA-HPPS group, whereas the signals within the LNs were very weak. Interestingly, the opposite trend was seen in T-MLNs, which had strong PA signals within the SLNs (Fig. 8a, and Movies S1-S3). Although the PA signals of the LNs in the HPPS and 5K-HA-e groups exhibited the same trend in terms of distribution as T-MLNs in 5K-HA-HPPS, their signal intensities were much weaker (Fig. 8a). To quantitatively analyse the distribution characteristics of the PA signals in LNs with different statuses, we extracted PA signals along the transverse (yellow line) and longitudinal (white line) diameters of each LN and drew crosssectional intensity profiles (Fig. 8b). The diameters of the LNs with differing statuses were normalised, and the centre of each LN was denoted as the zero position (Supplementary Fig. S9). The ratios ( $R$ values) of PA 


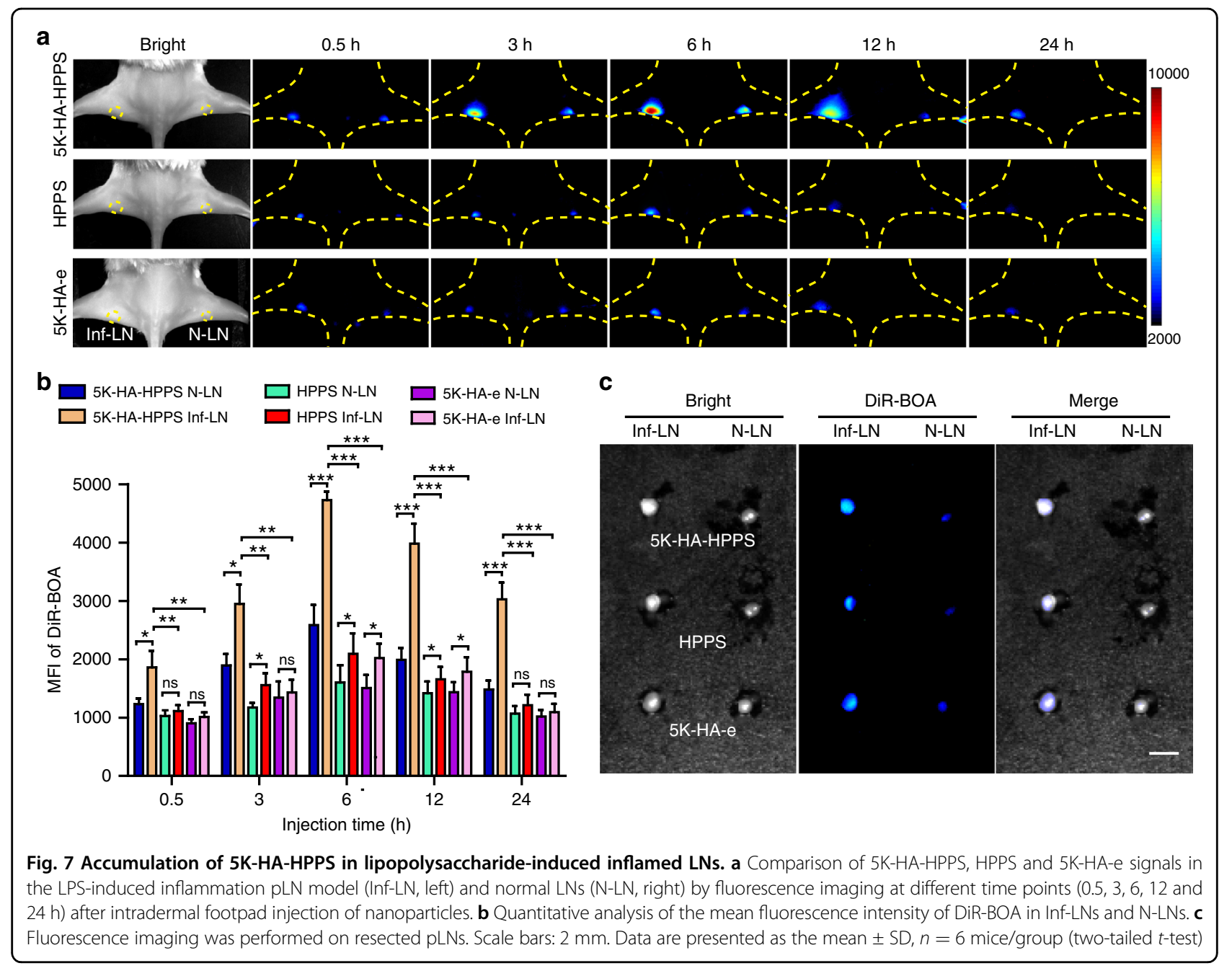

intensities at the centre of the LNs to those at their peripheries were $5.93 \pm 0.75$ for T-MLNs of the 5K-HAHPPS group, which was much higher than that for InfLNs $\quad(R=0.2 \pm 0.07)$ and N-LNs $(R=0.45 \pm 0.09)$. Although the intensity profiles of the HPPS and 5K-HA-e groups were similar to that of the 5K-HA-HPPS group, the ratios of PA intensity for T-MLNs of the HPPS ( $R=$ $3.06 \pm 0.4)$ and $5 \mathrm{~K}-\mathrm{HA}-\mathrm{e}(R=3.05 \pm 0.5)$ groups were $\sim 50 \%$ of that of the $5 \mathrm{~K}-\mathrm{HA}$-HPPS group $(R=5.93 \pm 0.75)$ (Fig. 8c). The characteristic distribution patterns of nanoparticles in the LNs with different statuses were also confirmed by confocal microscopy (Fig. 8d). Moreover, we performed PAM on earlier T-MLNs at 1 and 2 weeks after tumour inoculation, and distribution of the PA signals was found to concur with those SLNs at 3 weeks of metastasis (Supplementary Fig. S10). Thus, 5K-HA-HPPS combined with PAM can not only distinguish T-MLNs from Inf-LNs and N-LNs but can also be applied to detect early T-MLNs, guiding clinicians in removing SLNs during breast cancer surgery.

\section{Discussion}

LNs are among the most important organs of the immune system and play critical roles in not only removing pathogens but also producing lymphocytes and antibodies. SLNs are LNs in which a primary tumour first metastasises. Accurate detection and characterisation of SLNs are crucial for cancer staging and making therapeutic decisions, particularly in patients with breast cancer or melanomas ${ }^{32,33}$. At present, the clinical gold standard used to detect SLNs is labelling with blue dye or a radioactive nanocolloid and then performing SLNB. Although various nanoprobes based on different imaging modalities have been developed to map the metastatic status of SLNs ${ }^{12-15,34-36}$, few can distinguish T-MLNs from Inf-LNs. Tumour cell infiltration and proliferation of immune cells due to the induction of inflammatory factors result in the enlargement of SLNs ${ }^{37}$, usually leading to misidentification and unnecessary removal of benign SLNs during surgical resection ${ }^{38}$. Therefore, whether SLN enlargement is caused by the metastasis of tumour cells or 


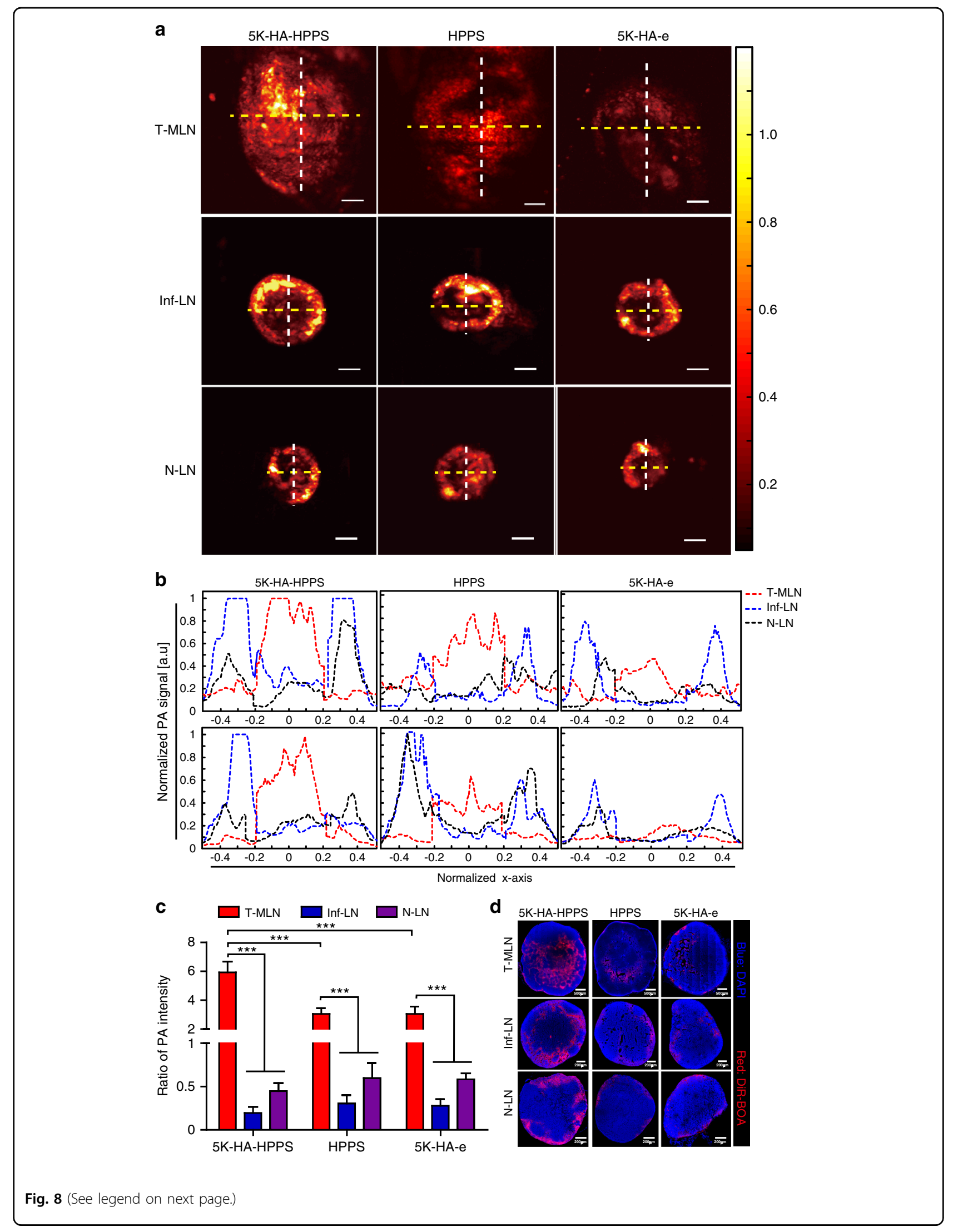


(see figure on previous page)

Fig. 8 In vivo PAM of 5K-HA-HPPS in LNs with different statuses. a In vivo comparison of the distribution of 5K-HA-HPPS (left), HPPS (middle) and 5K-HA-e (right) in 4T1-related SLNs (upper), inflamed LNs (middle) and normal LNs (lower) obtained using the AR-PAM system at $6 \mathrm{~h}$ after intratumoural injection (left) or footpad injection (right). Scale bar: $500 \mu \mathrm{m} . n=6$ per group. $\mathbf{b}$ Normalised PA signals of 5K-HA-HPPS, HPPS and 5KHA-e for distribution and intensity profiles that were taken from the transverse (yellow line) and longitudinal (white line) diameters of each LN depicted in $\mathbf{a}$. To quantitatively analyse the distribution of PA signals, the diameters of the LNs with different statuses were normalised, and their centres were designated as the zero position. c The ratio of PA intensities ( $R$ values) at the centre of the LNs to those at their periphery. Data are presented as the mean $\pm S D, n=6$ mice/group (two-tailed $t$-test). $\mathbf{d}$ After in vivo PAM of LNs with different statuses, frozen sections were taken and confocal microscopy was performed. Blue: DAPI, red: DiR-BOA. Slices: $10 \mu \mathrm{m}$

inflammation should be determined before SLN resection to reduce potential complications caused by unnecessary resection.

Breast cancer cells are well known to overexpress CD44 proteins. Recently, Tao et al. reported that SR-B1 is also expressed on breast cancer cells ${ }^{26}$. Previously, we developed an HPPS nanoparticle with strong SR-B1 targeting ability $^{24}$. By incorporating HA into HPPS, we established a novel dual-targeting 5K-HA-HPPS that can simultaneously target CD44 and SR-B1. It is well understood that lymphatic endothelial cells in LNs express lymphatic vessel endothelial hyaluronan receptor 1 (Lyve-1), which is a homologue of the CD44 glycoprotein and a lymphspecific receptor that specifically binds $\mathrm{HA}^{39,40}$. Yang et al. used HA of different molecular weights $(5 \mathrm{~K} / 10 \mathrm{~K} / 15 \mathrm{~K})$ as SLN mapping agents and reported that $10 \mathrm{~K}$ HA had favourable migration and retention profiles because it binds to Lyve-1 in LNs. The combination of HA and tumour-targeting antibodies (EGFR and HER2) can detect UM-SCC-22B tumour metastasis in SLNs ${ }^{41}$. However, this method of identifying T-MLNs is not ideal because it can only identify partial and fully occupied SLNs due to the minimal blood supply able to deliver the imaging probes to micrometastases and the simultaneous injection of antibody and HA, which should be separated by $24 \mathrm{~h}$. In our study, we found that 5K-HA-HPPS rapidly $(<10 \mathrm{~min})$ migrated to pLNs and showed prolonged retention (>12 h) compared with 15K-HA-HPPS. Indeed, fluorescence imaging of LN sections demonstrated that 5K-HA-HPPS can partially bind Lyve-1 within LNs (Supplementary Fig. S11). In addition to 4T1 cells, we demonstrated that 5K-HA-HPPS has the same targeting effect in B16F10 melanoma cells and CT26 colorectal cancer cells (Supplementary Fig. S7), suggesting that dualtargeted 5K-HA-HPPS can be applied to a variety of tumour cells. Due to the excellent scalability of the nanocarrier HPPS, it can be loaded with different imaging contrast agents to detect the migration of immune cells ${ }^{42}$ and can also carry drugs (e.g. curcumin ${ }^{43}$ ) for experimental autoimmune encephalomyelitis treatment or carry immune adjuvants (e.g. $\mathrm{CpG}^{21}$ ) for tumour treatment. In this study, the nanoparticles were loaded with molecular HA. We can also change the targeting ligand according to different biomarkers (e.g. EGFR, folate receptor) expressed by tumours ${ }^{24}$. Therefore, this novel nanoparticle probe may provide better diagnostic imaging and therapeutic potential for the detection and treatment of serious diseases.

LN enlargement can occur during both tumour cell invasion and under inflammatory conditions. Recently, various imaging agents have been developed to identify the metastatic status of SLNs based on indirect mapping agents such as single-walled carbon nanotubes ${ }^{44}$, albu$\min ^{45}$ and upconversion nanoparticles ${ }^{46}$. The use of these indirect mapping agents is not the best means to distinguish T-MLNs because it is difficult to detect SLNs that are completely occupied by tumour cells. Hence, the ideal mapping agent can directly target tumour cells and distinguish T-MLNs from Inf-LNs. In our study, the fluorescence signal of 5K-HA-HPPS in enlarged Inf-LNs was enhanced compared with that in N-LNs. This may be attributed to the proliferation of macrophages and lymphangiogenesis caused by inflammatory stimulation, thus resulting in enhanced accumulation of 5K-HA-HPPS in Inf-LNs. Fluorescence imaging showed that 5K-HA-HPPS accumulated in both T-MLNs and Inf-LNs (Figs. 6, 7), indicating that T-MLNs and Inf-LNs cannot be distinguished according to their fluorescence intensities. However, we found that 5K-HA-HPPS can effectively target 4T1 cells in metastatic LNs.

PAM is a rapidly developing imaging technology, and all types of nanoscale agents have been comprehensively explored as PA imaging probes to map SLNs ${ }^{34,47-49}$. Luke et al. introduced EGFR-targeted plasmonic nanosensors (MAPS), which can noninvasively and effectively identify oral squamous cell carcinoma sLN micrometastases from normal LNs by using ultrasound-guided spectroscopic photoacoustic (sPA) imaging ${ }^{50}$. This method has great potential for clinical translation due to combined ultrasound imaging, sPA imaging and MAPS. Previously, we developed a self-assembled nano-pomegranate with the NIR dye DiR-BOA, which has fluorescence/photoacoustic imaging-switching ('off/on') capability ${ }^{28}$. Therefore, we attempted to distinguish the different statuses of LNs using PAM. Remarkably, we found that PA signals from 5K-HA-HPPS showed a significantly distinct spatial distribution among LNs of different statuses. This result suggests that 5K-HA-HPPS possesses a potent ability to 
distinguish T-MLNs from Inf-LNs and N-LNs. The potential reason for the spatial differences of 5K-HAHPPS in T-MLNs and Inf-LNs is the difference in targeting efficiencies between tumour cells and macrophages. Our results showed that 5K-HA-HPPS is mainly taken up by macrophages in inf-LNs and partially by LECs and DCs (Supplementary Fig. S8b), while it more effectively targets metastatic $4 \mathrm{~T} 1$ cells in the inner part of TMLNs than cells in the peripheral region of T-MLNs in vivo (Fig. 5d). The PA signal of 5K-HA-HPPS was weak in early T-MLNs (1-2 weeks) (Supplementary Fig. S10), perhaps because fewer tumour cells metastasised into SLNs, but the distribution was still different from that in Inf-LNs and N-LNs.

In summary, we developed a novel dual-targeting agent, 5K-HA-HPPS, with fluorescence/photoacoustic dualmodal imaging capability. Wide-field fluorescence imaging showed that 5K-HA-HPPS can effectively map pLNs and that the fluorescence intensities of the nanoparticles were enhanced in T-MLNs and Inf-LNs. Surprisingly, PA showed a reverse spatial distribution in LNs with different statuses, whereby the PA signals of 5K-HA-HPPS were mainly distributed within T-MLNs but at the peripheries of Inf-LNs and N-LNs because 5K-HA-HPPS can effectively target metastatic tumour cells in T-MLNs. Thus, 5K-HA-HPPS can provide important guidance for the resection of SLNs in breast cancer surgery and facilitate the implementation of appropriate tumour treatment strategies.

\section{Materials and methods}

1,2-Dimyristoyl-sn-glycero-3-phosphocholine (DMPC), 1,2-dimyristoyl-sn-glycero-3-phosphoethanolamine (DMPE) and 1,2-distearoyl-sn-glycero-3-phosphoethanolamine-N[methoxy(polyethylene glycol)-2000] (ammonium salt) (DSPE-PEG2000, ammonium salt) were obtained from Avanti Polar Lipids Inc. (Alabaster, AL, USA). HA with different molecular weights of 5 and $15 \mathrm{kDa}$ were obtained from Lifecore Biomedical LLC (MN, USA) (cat\#: HA5K-1, HA15K-1). 1-Ethyl-3-[3-dimethyl)aminopropyl]carbodiimide (EDAC) was purchased from Sigma-Aldrich Co. (St. Louis, MO, USA). The near-infra-red dye DiR-BOA (1,1'-dioctadecyl-3,3,3',3'-tetra-methylindotricarbocyanine iodide bisoleate) was synthesised as previously described ${ }^{51}$. R4F (an ApoA1-mimetic peptide, Ac-FAEKFKEAVKDYFAKFWD) was synthesised by Shanghai Apeptide Co., Ltd. (Shanghai, China).

\section{Mice and cells}

Albino-C57BL/6 transgenic mice were obtained from the Jackson Laboratory (Bar Harbor, ME). BALB/c female mice were purchased from the Hubei Research Center of Laboratory Animals (Hubei, China). All animal studies were conducted in compliance with protocols that had been approved by the Hubei Provincial Animal Care and Use Committee and in compliance with the experimental guidelines of the Animal Experimentation Ethics Committee of Huazhong University of Science and Technology (IACUC number: 2160). The mouse mammary adenocarcinoma 4T1 cell line was kindly provided by Professor Li Su (Huazhong University of Science and Technology). 4T1-tfRFP cells were obtained by transfecting 4T1 cells with a plasmid containing the tfRFP gene ${ }^{52}$. The B16F10 cell line was purchased from BOSTER Company (Wuhan, China), and the CT26 cell line was obtained from the American Type Culture Collection (Manassas, USA). These cells were cultured in complete RPMI-1640 medium (Gibco, Thermo Fisher Scientific, USA) containing $10 \%$ foetal bovine serum (FBS, Gibco) and $1 \%$ penicillin-streptomycin (Gibco) in a cell incubator (Thermo, USA) with $5 \% \mathrm{CO}_{2}$ and $95 \%$ air at $37^{\circ} \mathrm{C}$.

\section{Synthesis of HA-DMPE}

HA-DMPE was synthesised as described previously using a modified reaction ${ }^{53}$. Briefly, $14 \mathrm{mg}$ of $\mathrm{HA}$ was dissolved in $5 \mathrm{ml}$ of distilled water, and $6 \mathrm{mg}$ of EDAC was added at $\mathrm{pH} 4$ to preactivate the HA $(5$ and $15 \mathrm{kDa})$ for $2 \mathrm{~h}$ at $37^{\circ} \mathrm{C}$. Subsequently, a suspension of DMPE $(0.5 \mathrm{mg})$ was added to the preactivated HA solution, and the $\mathrm{pH}$ was adjusted to 8.6 with $0.1 \mathrm{M}$ borate buffer $(\mathrm{pH}$ 9.4). This reaction proceeded for $24 \mathrm{~h}$ at $37^{\circ} \mathrm{C}$. The mixture was purified by dialysis (MWCO $3 \mathrm{kDa}$ ) four times for $12 \mathrm{~h}$ each time. Then, the purified solution was lyophilised, and $1 \mathrm{ml}$ of chloroform-methanol $(4: 1 ; \mathrm{v} / \mathrm{v})$ was added to the lyophilised sample. Next, the sample was centrifuged at $12,000 \mathrm{rpm}$ for $10 \mathrm{~min}$ at $4{ }^{\circ} \mathrm{C}$; this step was repeated three times. The free HA precipitate was removed, and the supernatant of the HA-DMPE solution was collected and stored at $4{ }^{\circ} \mathrm{C}$. The structures of HA, DMPE and the HA-DMPE copolymers were determined using ${ }^{1} \mathrm{H}$ NMR (AV400, Bruker, Switzerland) in $\mathrm{D}_{2} \mathrm{O}$ and DMSO- $d_{6}$.

\section{Synthesis and characterization of the HA-HPPS core loaded with DiR-BOA}

HA-HPPS complexes were synthesised as follows. DMPC $(3 \mu \mathrm{mol})$, cholesterol oleate $(\mathrm{CO}, 0.1 \mu \mathrm{mol})$, DiRBOA $(0.2 \mu \mathrm{mol})$, DSPE-PEG2000 $(0.0114 \mu \mathrm{mol})$ and HADMPE $(5$ or $15 \mathrm{kDa}, 0.04 \mu \mathrm{mol})$ in chloroform $(400 \mu \mathrm{l})$ were dried under a nitrogen stream to form a uniform film. Then, $2 \mathrm{ml}$ of PBS solution was added to the dried film and vortexed for $5 \mathrm{~min}$. Subsequently, the mixture was sonicated for $\sim 1 \mathrm{~h}$ at $48^{\circ} \mathrm{C}$. $\mathrm{R} 4 \mathrm{~F}(0.78 \mu \mathrm{mol})$ was dissolved in $1 \mathrm{ml}$ of PBS, added dropwise to the lipid emulsion and stored overnight at $4{ }^{\circ} \mathrm{C}$. After concentration using centrifugal filter units (30 kDa, Millipore, USA), the nanoparticles were purified using the Akta FPLC system with a HiLoad 16/70 Superose 6 column (General 
Electric Healthcare, NY, USA). The peptide concentration was measured using a CBQCA protein quantitation kit (Invitrogen Corporation, CA, USA). The morphologies of the nanoparticles were analysed by TEM (TECNAI G2, FEI Company, OR, USA). The size distributions of the nanoparticles were measured by DLS on a Zetasizer Nano-ZS90 (Malvern Instruments, Worcestershire, UK). The stability of the nanoparticles was evaluated using seminative SDS-PAGE.

\section{Cytotoxicity test}

The cytotoxicity of the samples to $4 \mathrm{~T} 1$ cells was determined by the MTS assay. In brief, $1 \times 10^{4} 4 \mathrm{~T} 1$ and RAW264.7 cells (in $100 \mu \mathrm{l}$ of medium) were seeded in each well of a 96-well culture plate and incubated at $37^{\circ} \mathrm{C}$ with $5 \% \mathrm{CO}_{2}$ for $24 \mathrm{~h}$. The medium in each well was replaced with culture medium $(100 \mu \mathrm{l})$ containing different concentrations $(1-40 \mu \mathrm{M})$ of HA-HPPS. After incubation for $24 \mathrm{~h}, 20 \mathrm{\mu l}$ of MTS was added to each well, and incubation was continued for $1 \mathrm{~h}$ at $37^{\circ} \mathrm{C}$. Then, absorption at $490 \mathrm{~nm}$ was measured using a Bio-Tek Epoch microplate spectrophotometer (Winooski, Vermont, USA).

\section{Confocal imaging}

To illustrate the 4T1 cell dual-targeting ability of the nanoparticles in vitro, 4T1 cells, RAW264.7 cells, BMDMs and BMDCs were seeded into 8-well chambers covering the glass bottoms (Nunc Lab-Tek, Thermo Scientific) $\left(2 \times 10^{4}\right.$ cells/well $)$. Then, the cells were incubated with $5 \mathrm{~K}-\mathrm{HA}-\mathrm{HPPS}$, HPPS or $5 \mathrm{~K}-\mathrm{HA}-\mathrm{e}$ at a DiR-BOA concentration of $10 \mu \mathrm{M}$ for $3 \mathrm{~h}$, and Hoechst $33258\left(0.5 \mu \mathrm{g} \mathrm{ml}^{-1}\right)$ was added $15 \mathrm{~min}$ before washing. For imaging of the tissue sections, the LNs were fixed in $4 \%$ paraformaldehyde for $10 \mathrm{~h}$ at $4{ }^{\circ} \mathrm{C}$ and then dehydrated in a $30 \%$ sucrose solution. The LNs were then frozen in OCT compound (Sakura, Torrance, CA, USA) and sectioned into $10-\mu \mathrm{m}$-thick slices using a freezing microtome (Leica, Germany). For staining, slides were washed once with PBS, and the sections were immunostained with Lyve-1 (eBioscience, clone ALY7, 1:200), F4/80 (Clone: BM8, 1:200), CD11c (Clone: N418, 1:100), CD3 (Clone: 17A2, 1:200) and B220 (Clone: RA3-6B2, 1:200). Fluorescence images were acquired using an LSM 710 laser confocal scanning microscope (Zeiss, Germany) with an excitation wavelength of $405 \mathrm{~nm}$ for Hoechst 33258 and DAPI, $488 \mathrm{~nm}$ for FITC and Lyve-1 and $633 \mathrm{~nm}$ for DiR-BOA.

\section{FCM analysis}

For 4T1 cell-targeting ability testing, 4T1 cells, B16 cells, CT26 cells and E0771 cells were plated in 96-well flat-bottom culture plates $\left(5 \times 10^{4}\right.$ cells/well $)$, and $5 \mathrm{~K}$-HAHPPS, HPPS or 5K-HA-e were incubated with the cells at various DiR-BOA concentrations for 0.5 or $3 \mathrm{~h}$. Regarding the competition assay, 4T1 cells were precultured with excessive amounts of HDL protein and free HA. The mass ratio of HDL to R4F in 5K-HA-HPPS and HPPS was 10:1 and that of free HA to HA in 5K-HA-HPPS and 5K-HA-e was 250:1. Then, the cells were incubated with 5K-HAHPPS, HPPS or 5K-HA-e at a DiR-BOA concentration of $10 \mu \mathrm{M}$ for $3 \mathrm{~h}$. The total volume of the solution in the wells was $200 \mu \mathrm{l}$ for FCM. Fluorescence signals were quantified by a Guava easyCyte $8 \mathrm{HT}$ flow cytometer (Millipore Corporation, Billerica, MA, USA). The data were analysed using FlowJo.

\section{LN metastasis and inflammation models}

To establish the LN metastasis model of 4T1 murine breast cancer, $5 \times 10^{5} 4 \mathrm{~T} 1$ cells or $4 \mathrm{~T} 1$-tfRFP cells in $20 \mu \mathrm{l}$ of PBS were injected into the left hock area of BALB/c mice. Albino-C57BL/6 mice were used to develop the pLN inflammation model by intra-lymph node injection of LPS ( $5 \mathrm{ml} \mathrm{kg}^{-1}$, Sigma) 2 days before imaging experiments ${ }^{11}$. In vivo imaging experiments were performed using a custom-made whole-body optical imaging system.

\section{In vivo fluorescence imaging}

For in vivo fluorescence imaging of the LNs, $25 \mathrm{nmol}$ of $5 \mathrm{~K}-\mathrm{HA}-\mathrm{HPPS}$, HPPS or $5 \mathrm{~K} / 15 \mathrm{~K}$-HA-e were injected into one side of the footpad of normal Albino-C57BL/6 mice, and nanoparticle accumulation in pLNs and sLNs was detected and analysed by wide-field fluorescence imaging at $10 \mathrm{~min}, 1,3,6$ and $12 \mathrm{~h}$ after injection of the probe. For fluorescence imaging of Inf-LNs and TMLNs, 5K-HA-HPPS, HPPS or 5K/15K-HA-e were injected into both sides of the footpad of inflammatory Albino-C57BL/6 mice and the hock area of BALB/c mice. The fluorescence signals of N-LNs, T-MLNs and Inf-LNs were imaged at $0.5,3,6,12$ and $24 \mathrm{~h}$ after injection of the probes. Fluorescence images of DiRBOA were acquired with an NIR filter set (excitation: $716 / 40 \mathrm{~nm}$; emission: $800 / 40 \mathrm{~nm}$; exposure time: 10 or $30 \mathrm{~s})$. The mice were anaesthetised with $3 \%$ isoflurane/ $\mathrm{O}_{2}(\mathrm{v} / \mathrm{v})$ and maintained on isoflurane $/ \mathrm{O}_{2}$ at $1.5 \%(\mathrm{v} / \mathrm{v})$ throughout the experiments. For LN imaging, 3 weeks after hock inoculation of 4T1-tfRFP tumour cells, resected T-MLNs were imaged $6 \mathrm{~h}$ after intratumoural injection of FITC-5K-HA-HPPS and FITC-HPPS. Fluorescence images of tfRFP and FITC were acquired with a filter set (excitation: 562/40 nm, emission: 640/ $40 \mathrm{~nm}$; and excitation: $496 / 40 \mathrm{~nm}$, emission: 562/ $40 \mathrm{~nm}$, respectively). Regarding the orthotropic breast cancer model, tumour-bearing mice were subjected to fluorescence imaging on day 30 after tumour inoculation $(n=3)$. A single dose of 5K-HA-HPPS or HPPS (DiR-BOA: $25 \mathrm{nmol}$ ) in $50 \mu \mathrm{l}$ of sterile PBS was injected intratumourally. Imaging of brachial and axillary LNs was performed $6 \mathrm{~h}$ after injection of the probes. 


\section{In vitro and in vivo PAM}

For in vitro PAM of 4T1 cells and BMDMs, 4T1 cells and BMDMs were seeded into $15 \mathrm{~mm}$ dishes and incubated with 5K-HA-HPPS, HPPS or 5K-HA-e for $3 \mathrm{~h}$ in vitro.

For in vivo PAM analysis of different statuses of LNs, the skin was excised and pLNs were exposed $6 \mathrm{~h}$ after injection of 5K-HA-HPPS, HPPS or 5K-HA-e (DiR-BOA: $25 \mathrm{nmol}$ ). Photoacoustic (PA) maximum amplitude projection images (image field of view: $5 \mathrm{~mm} \times 5 \mathrm{~mm}$ ) were taken in vivo with the custom-made acoustic resolution PAM (AR-PAM) system with $744 \mathrm{~nm}$ pulsed lasers at $600 \mathrm{~nJ}$. The images were acquired by performing a B-scan, with each B-scan including 500 steps with a step size of $10 \mu \mathrm{m}$. The maximum image depth of AR-PAM was $1.6 \mathrm{~mm}$. The spatial resolution of ARPAM was $\sim 45 \mu \mathrm{m}$. To quantitatively analyse the distribution of PA signals in LNs with different statuses, we extracted PA signals along the transverse (dotted yellow line) and longitudinal (dotted white line) diameters of each LN and constructed cross-sectional intensity profiles to reduce error from single section measurement. The $\mathrm{LN}$ centre (C) was defined as $40 \%$ of the diameter $(20 \%$ on each side of the centre point). The LN periphery ( $\mathrm{P}$ ) was defined as $60 \%$ of the diameter ( $30 \%$ on each side of the edge). The average ratio of PA intensity in the $\mathrm{LN}$ centre $(\mathrm{C})$ to that of the $\mathrm{LN}$ periphery (P) was calculated by the formula $R=\left(R_{\mathrm{T}}+R_{\mathrm{L}}\right) / 2$. $R_{\mathrm{T}}$ and $R_{\mathrm{L}}$ represent the ratio of transverse $(\mathrm{T})$ and longitudinal (L) PA intensity, respectively, and were calculated as follows:

$$
\begin{aligned}
& R_{T}=C_{1} /\left(\frac{P_{1}+P_{2}}{2}\right), \\
& R_{L}=C_{2} /\left(\frac{P_{3}+P_{4}}{2}\right),
\end{aligned}
$$

where $C_{1}$ and $C_{2}$ represent the PA intensities of the $\mathrm{LN}$ centre in the transverse and longitudinal sections, respectively; $P_{1}$ and $P_{2}$ represent the PA intensities of the LN periphery in the transverse section on the left and right sides, respectively; and $P_{3}$ and $P_{4}$ represent the PA intensities of the LN periphery in the longitudinal section on the top and bottom, respectively (Supplementary Fig. S9).

\section{Statistical analysis}

Statistical analysis was performed using GraphPad Prism 5 (GraphPad Software, CA). Student's $t$ test (two tailed) was used for the in vitro and in vivo studies. Data are presented as the mean \pm SD. Significant differences between or among the groups are indicated as follows: ns for no significant difference, " for $P<0.05$, *** for $P<0.01$, and ${ }^{* * * *}$ for $P<0.001$.

\section{Acknowledgements}

We thank the Optical Bioimaging Core Facility of WNLO-HUST for support in data acquisition, the Center for Nanoscale Characterization \& Devices (CNCD, Tecnai $\mathrm{G}^{2} 20$ U-Twin) of WNLO-HUST for facility support, and the Analytical and
Testing Center of HUST for ${ }^{1} \mathrm{H}$ NMR measurements. This work was supported by the National Science Fund for Distinguished Young Scholars (81625012), the National Natural Science Foundation of China (61721092), the Fundamental Research Funds for the Central Universities (2019kfyXMBZ022), Programme for HUST Academic Frontier Youth Team (Zhang, Z.H.), and the Innovation Fund of WNLO.

\section{Author contributions}

Y.D., X.Y. and J.W., under the direction of Z.Z., designed and performed the experiments, analysed the data and wrote the first version of the manuscript. J.W F.Z. and Y.L performed part of the experiments. Q.L. and Z.Z. designed the experiments and supervised the study. Z.Z. wrote the manuscript. All authors contributed to the critical revision of the manuscript and approved the final version.

\section{Conflict of interest}

The authors declare that they have no conflict of interest.

Supplementary information is available for this paper at https://doi.org/ 10.1038/s41377-020-00399-0.

Received: 3 May 2020 Revised: 14 August 2020 Accepted: 1 September 2020

Published online: 16 September 2020

\section{References}

1. Siegel, R. L., Miller, K. D. \& Jemal, A. Cancer statistics, 2019. CA: Cancer J. Clin. 69 7-34 (2019).

2. Bray, F. et al. Global cancer statistics 2018: GLOBOCAN estimates of incidence and mortality worldwide for 36 cancers in 185 countries. CA: A Cancer J. Clin. 68, 394-424 (2018).

3. Mehta, R. S. et al. Overall survival with fulvestrant plus anastrozole in metastatic breast cancer. N. Engl. J. Med. 380, 1226-1234 (2019).

4. McGuire, A., Brown, J. A. L. \& Kerin, M. J. Metastatic breast cancer: the potential of miRNA for diagnosis and treatment monitoring. Cancer Metastasis Rev. 34, 145-155 (2015).

5. Hayes, D. F. HER2 and breast cancer-a phenomenal success story. N. Engl. J. Med. 381, 1284-1286 (2019).

6. Lowes, S. et al. Evolving imaging techniques for staging axillary lymph nodes in breast cancer. Clin. Radiol. 73, 396-409 (2018).

7. Veronesi, $U$. et al. Sentinel lymph node biopsy in breast cancer: ten-year results of a randomized controlled study. Ann. Surg. 251, 595-600 (2010).

8. Ahmed, M., Purushotham, A. D. \& Douek, M. Novel techniques for sentinel lymph node biopsy in breast cancer: a systematic review. Lancet Oncol. 15, e351-e362 (2014)

9. Soares, E. W. S. et al. Morbidity after conventional dissection of axillary lymph nodes in breast cancer patients. World J. Surg. Oncol. 12, 67 (2014).

10. Bello, M. A. et al. Incidence complications following sentinel lymph node biopsy or axillary lymph node dissection after breast cancer surgery. J. Clin. Oncol. 30, 97 (2012)

11. Yoo, J. S. et al. A macrophage-specific fluorescent probe for intraoperative lymph node staging. Cancer Res. 74, 44-55 (2014).

12. Liu, J. et al. Percutaneous contrast-enhanced ultrasound for localization and diagnosis of sentinel lymph node in early breast cancer. Sci. Rep. 9, 13545 (2019).

13. Zhou, Z. J. et al. A protein-corona-free $T_{1}-T_{2}$ dual-modal contrast agent for accurate imaging of lymphatic tumor metastasis. ACS Appl. Mater. Interfaces 7, 28286-28293 (2015).

14. Zhang, F. et al. Differentiation of reactive and tumor metastatic lymph nodes with diffusion-weighted and SPIO-enhanced MRI. Mol. Imaging Biol. 15, 40-47 (2013).

15. Sun, P. P. et al. Image-guided surgery of head and neck carcinoma in rabbit models by intra-operatively defining tumour-infiltrated margins and metastatic lymph nodes. EBioMedicine 50, 93-102 (2019).

16. Toole, B. P. Hyaluronan: from extracellular glue to pericellular cue. Nat. Rev. Cancer 4, 528-539 (2004).

17. Burdick, J. A. \& Prestwich, G. D. Hyaluronic acid hydrogels for biomedical applications. Adv. Mater. 23, H41-H56 (2011). 
18. Götte, M. \& Yip, G. W. Heparanase, hyaluronan, and CD44 in cancers: a breast carcinoma perspective. Cancer Res. 66, 10233-10237 (2006).

19. Aguiar, D. J., Knudson, W. \& Knudson, C. B. Internalization of the hyaluronan receptor CD44 by chondrocytes. Exp. Cell Res. 252, 292-302 (1999).

20. Katoh, S. et al. Glycosylation of CD44 negatively regulates its recognition of hyaluronan. J. Exp. Med. 182, 419-429 (1995).

21. Qian, Y. et al. Targeting dendritic cells in lymph node with an antigen peptidebased nanovaccine for cancer immunotherapy. Biomaterials 98, 171-183 (2016).

22. Hameed, S. et al. Fluorescence guided sentinel lymph node mapping: from current molecular probes to future multimodal nanoprobes. Bioconjugate Chem. 30, 13-28 (2019).

23. Trevaskis, N. L., Kaminskas, L. M. \& Porter, C. J. H. From sewer to saviourtargeting the lymphatic system to promote drug exposure and activity. Nat. Rev. Drug Discov. 14, 781-803 (2015).

24. Zhang, Z. H. et al. HDL-mimicking peptide-lipid nanoparticles with improved tumor targeting. Small 6, 430-437 (2010).

25. Yu, X. et al. Melittin-lipid nanoparticles target to lymph nodes and elicit a systemic anti-tumor immune response. Nat. Commun. 11, 1110 (2020).

26. Tan, T. et al. Bioinspired lipoproteins-mediated photothermia remodels tumor stroma to improve cancer cell accessibility of second nanoparticles. Nat. Commun. 10, 3322 (2019).

27. $\mathrm{Yu}, \mathrm{X}$. et al. Immune modulation of liver sinusoidal endothelial cells by melittin nanoparticles suppresses liver metastasis. Nat. Commun. 10, 574 (2019).

28. Lin, Q. Y. et al. Self-assembled "Off/On" nanopomegranate for in vivo photoacoustic and fluorescence imaging: strategic arrangement of Kupffer cells in mouse hepatic lobules. ACS Nano 13, 1526-1537 (2019).

29. Fu, Q. R. et al. Photoacoustic imaging: contrast agents and their biomedical applications. Adv. Mater. 31, 1805875 (2019).

30. Men, X. J. \& Yuan, Z. Multifunctional conjugated polymer nanoparticles for photoacoustic-based multimodal imaging and cancer photothermal therapy. J. Innov. Optical Health Sci. 12, 1930001 (2019).

31. Seong, M. \& Chen, S. L. Recent advances toward clinical applications of photoacoustic microscopy: a review. Sci. Chin. Life Sci. https://doi.org/10.1007/ s11427-019-1628-7 (2020).

32. Coutant, C. et al. Comparison of models to predict nonsentinel lymph node status in breast cancer patients with metastatic sentinel lymph nodes: a prospective multicenter study. J. Clin. Oncol. 27, 2800-2808 (2009).

33. Faries, M. B. et al. Completion dissection or observation for sentinel-node metastasis in melanoma. N. Engl. J. Med. 376, 2211-2222 (2017).

34. $\mathrm{Wu}, \mathrm{Y}$. L. et al. A nanoprobe for diagnosing and mapping lymphatic metastasis of tumor using 3D multispectral optoacoustic tomography owing to aggregation/deaggregation induced spectral change. Adv. Funct. Mater. 29, 1807960 (2019)

35. Zang, J. et al. Combined ${ }^{68} \mathrm{Ga-NOTA-evans}$ blue lymphoscintigraphy and ${ }^{68} \mathrm{Ga}$-NOTA-RM26 PET/CT evaluation of sentinel lymph node metastasis in breast cancer patients. Bioconjugate Chem. 31, 396-403 (2020).
36. Tang, L. et al. Aptamer-functionalized, ultra-small, monodisperse silica nanoconjugates for targeted dual-modal imaging of lymph nodes with metastatic tumors. Angew. Chem. Int. Ed. 51, 12721-12726 (2012).

37. Wu, X. S. et al. Inflammation and tumor microenvironment in lymph node metastasis. Cancers 3, 927-944 (2011).

38. Giuliano, A. E. et al. Effect of axillary dissection vs no axillary dissection on 10year overall survival among women with invasive breast cancer and sentinel node metastasis: the ACOSOG Z0011 (Alliance) randomized clinical trial. JAMA 318, 918-926 (2017).

39. Rinaldi, E. \& Baggi, F. LYVE-1 is 'on stage' now: an emerging player in dendritic cell docking to lymphatic endothelial cells. Cell. Mol. Immunol. 15, 663-665 (2018).

40. Banerji, S. et al. LYVE-1, a new homologue of the CD44 glycoprotein, is a lymph-specific receptor for hyaluronan. J. Cell Biol. 144, 789-801 (1999).

41. Yang, X. Y. et al. Mapping sentinel lymph node metastasis by dual-probe optical imaging. Theranostics 7, 153-163 (2017).

42. Jin, H. L. et al. Magnetic enrichment of dendritic cell vaccine in lymph node with fluorescent-magnetic nanoparticles enhanced cancer immunotherapy. Theranostics 6, 2000-2014 (2016).

43. Lu, L. S. et al. Targeted immunomodulation of inflammatory monocytes across the blood-brain barrier by curcumin-loaded nanoparticles delays the progression of experimental autoimmune encephalomyelitis. Biomaterials $\mathbf{2 4 5}$, 119987 (2020).

44. Liang, C. et al. Tumor metastasis inhibition by imaging-guided photothermal therapy with single-walled carbon nanotubes. Adv. Mater. 26, 5646-5652 (2014).

45. Wang, Y. et al. In vivo albumin labeling and lymphatic imaging. Proc. Natl Acad. Sci. USA 112, 208-213 (2015).

46. Qiao, R. R. et al. Ultrasensitive in vivo detection of primary gastric tumor and lymphatic metastasis using upconversion nanoparticles. ACS Nano 9 2120-2129 (2015).

47. Akers, W. J. et al. Noninvasive photoacoustic and fluorescence sentinel lymph node identification using dye-loaded perfluorocarbon nanoparticles. ACS Nano 5, 173-182 (2011)

48. Pan, D. et al. Photoacoustic sentinel lymph node imaging with self-assembled copper neodecanoate nanoparticles. ACS Nano 6, 1260-1267 (2012).

49. Chen, $\mathrm{Q}$. et al. $\mathrm{H}_{2} \mathrm{O}_{2}$-responsive liposomal nanoprobe for photoacoustic inflammation imaging and tumor theranostics via in vivo chromogenic assay. Proc. Natl Acad. Sci. USA 114, 5343-5348 (2017).

50. Luke, G. P. et al. Sentinel lymph node biopsy revisited: ultrasound-guided photoacoustic detection of micrometastases using molecularly targeted plasmonic nanosensors. Cancer Res. 74, 5397-5408 (2014).

51. Corbin, I. R. et al. Enhanced cancer-targeted delivery using engineered highdensity lipoprotein-based nanocarriers. J. Biomed. Nanotechnol. 3, 367-376 (2007).

52. Luo, H. M. et al. Tetrameric far-red fluorescent protein as a scaffold to assemble an octavalent peptide nanoprobe for enhanced tumor targeting and intracellular uptake in vivo. FASEB J. 25, 1865-1873 (2011).

53. Surace, $C$. et al. Lipoplexes targeting the CD44 hyaluronic acid receptor for efficient transfection of breast cancer cells. Mol. Pharm. 6 1062-1073 (2009). 